\title{
Occult liver abscess following clinically unsuspected ingestion of foreign bodies
}

\author{
Ban CH Tsui MSc MD, J Mossey MD FRCSC
}

\begin{abstract}
BCH Tsui, J Mossey. Occult liver abscess following clinically unsuspected ingestion of foreign bodies. Can J Gastroenterol $1997 ; 11(5): 445-448$. Two uncommon cases of foreign body (a wooden clothespin and a toothpick) perforation of the gut with associated pyogenic liver abscesses are presented. These cases illustrate the difficulties of preoperative diagnosis. The lack of history of ingestion of foreign bodies, variable clinical presentation of the conditions and radiolucent natures of the foreign bodies all play a role in impeding the diagnosis preoperatively. This report emphasizes the role of ultrasound and computed tomographic scan in evaluating similar cases. Any patient with known risk factors for ingestion of foreign body should arouse suspicion and be investigated further.
\end{abstract}

Key Words: Computed tomographic scan, Foreign bodies, Liver abscess, Ultrasound

M ost ingested foreign bodies pass through the gastrointestinal tract without causing any intestinal damage or other complications. However, several reports have described sharp pointed objects, such as pins (1), fish bones (2) and wooden toothpicks $(3,4)$, perforating the gut and migrating to the liver, causing pyogenic liver abscess. According to our review, there has not been a reported case of pyogenic liver abscesses caused by a piece of blunt-ended wooden clothespin. In this report we examine two cases of foreign body (a wooden clothespin and a toothpick) perforation of the gut with associated pyogenic liver abscesses. The cases described here illustrate the difficulties of preoperative diagnosis and emphasize the need for attention towards this rare but serious problem.

\section{Abcès hépatique occulte après ingestion de corps étrangers}

RÉSUMÉ : On présente ici deux cas rares de perforation de l'intestin associés à l'ingestion de corps étrangers (pince à linge en bois et cure-dent) accompagnés d'abcès hépatiques pyogènes. Ces cas illustrent la difficulté de poser le diagnostic préopératoire compte tenu de l'absence d'antécédents d'ingestion de corps étrangers, de la variabilité des tableaux cliniques et des différentes propriétés radio-opaques des corps étrangers. Ce rapport souligne le rôle de l'échographie et de la tomodensitométrie pour l'évaluation de cas semblables. Tout patient chez qui on peut soupçonner l'ingestion de corps étrangers devrait éveiller les soupçons et subir les examens diagnostiques qui s'imposent.

\footnotetext{
Department of Anaesthesia, University of Alberta Hospitals; Department of Surgery, Royal Alexandra Hospital, Edmonton, Alberta Correspondence: Dr Ban CH Tsui, Department of Anaesthesia, University of Alberta Hospitals, 3B2.32 Walter C Mackenzie Health Sciences Centre, 8440-112 Street, Edmonton, Alberta T6G 2B7. Telephone 403-492-8861, fax 403-492-9610, e-mail btsui@pop.srv.ualberta.ca Received for publication March 24, 1997. Accepted May 2, 1997
} 


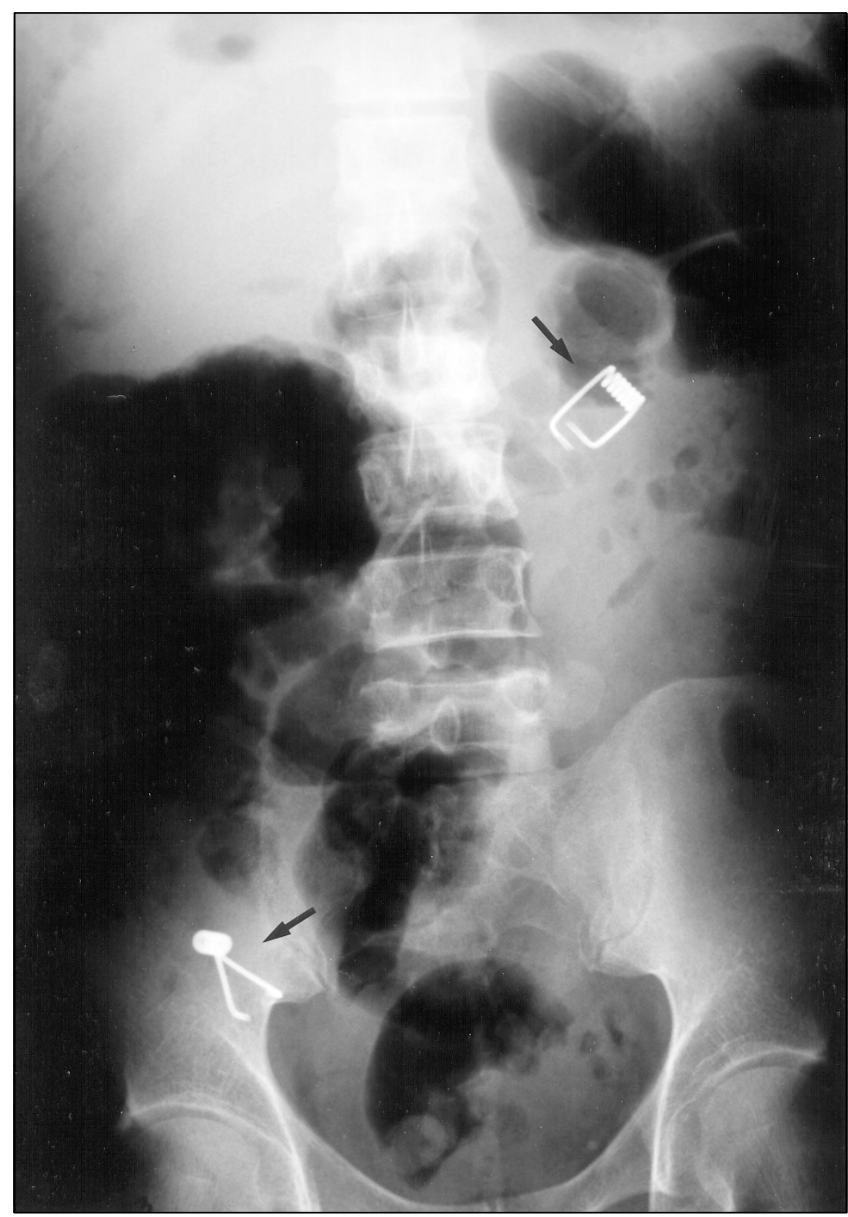

Figure 1) Case 1. Abdominal plain x-ray film shows two metallic foreign bodies in the upper left and lower right abdomen

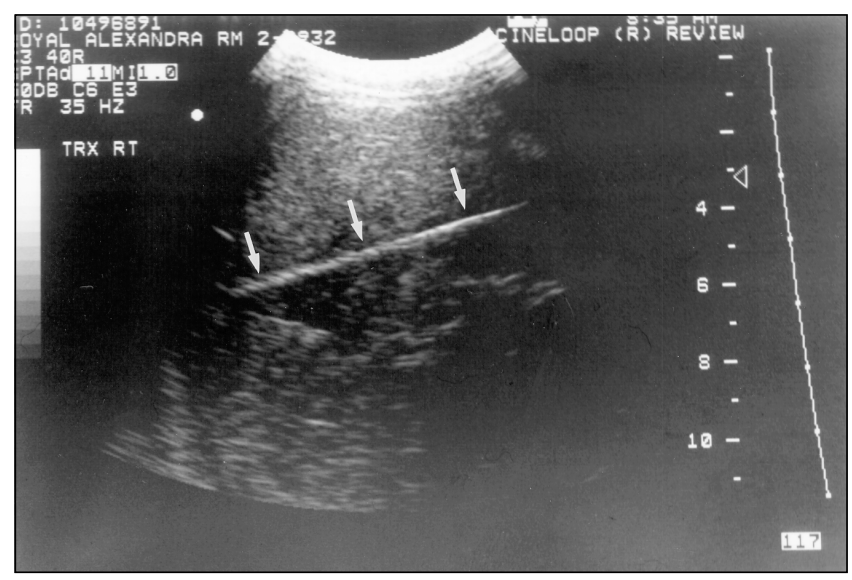

Figure 2) Case 1. Abdominal ultrasound shows a wooden clothespin (hyperechoic line) embedded in the liver abscess

phosphatase. Serum amylase was normal. Renal function studies were normal. Electrolytes showed hyponatremia of $129 \mathrm{mmol} / \mathrm{L}$. Abdominal plain films revealed multiple airfluid levels in the right upper quadrant. Two metallic foreign bodies (one in the left upper quadrant and the other in the right lower quadrant) were also identified (Figure 1). Chest

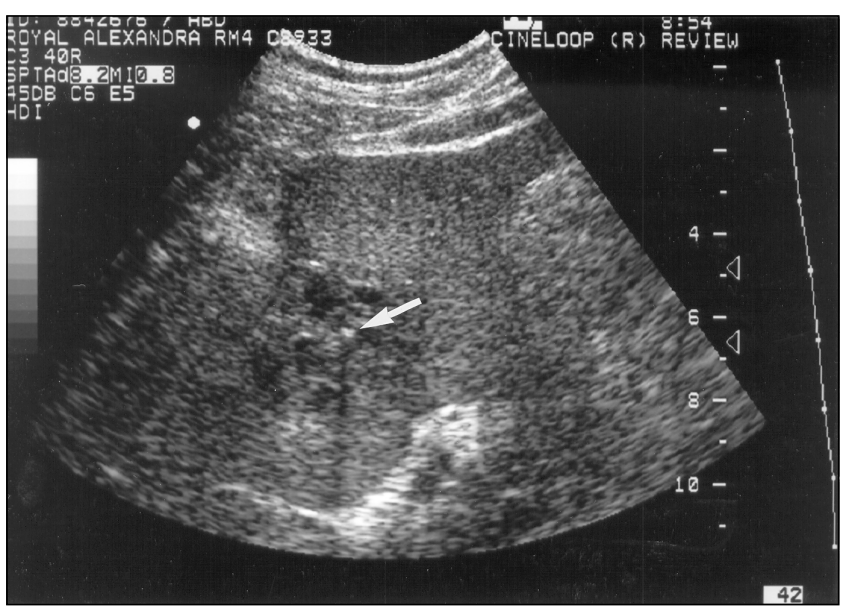

Figure 3) Case 2. Abdominal ultrasound shows a toothpick, seen as a tiny echogenic focus (arrow) in the liver abscess

$\mathrm{x}$-ray showed an elevated right hemidiaphragm, a small right pleural effusion and some right-sided atelectasis.

An exploratory laparotomy revealed dense adhesions with fibrin deposition in the right upper quadrant and a $500 \mathrm{~mL}$, foul-smelling subcapsular hematoma on the anterosuperior surface of the right lobe. The region was examined, debrided and drained. Multiple gastric foreign bodies were palpable in the stomach. Foreign bodies - including part of a clothespin, a piece of a leather belt and two pieces of plastic - were removed following gastrotomy. The small and large bowel were unremarkable, with no signs of perforation. Bacteriology studies on the subphrenic abscess revealed growth of Streptococci viridans. Intravenous antibiotics clindamycin and gentamicin were given postoperatively.

The patient tolerated the procedure well and was stable initially. However, he developed recurrence of right upper quadrant pain after one week. Abdominal ultrasound (Figure 2) and computed tomographic (CT) scan done later both showed the presence of an intrahepatic abscess and an unidentified foreign body. At second laparotomy, a gastrohepatic fistula from the lesser curve of the stomach to the left hepatic lobe was divided and oversewn. A wooden foreign body $(7 \times 0.6 \times 0.2 \mathrm{~cm})$, which resembled part of a clothespin, was removed from the intrahepatic abscess. The patient recovered from surgery uneventfully and was discharged home 10 days later.

Case 2: A 63-year-old edentulous man presented with a three-day history of severe right upper quadrant pain associated with nausea and vomiting. The pain was dull in character, but markedly increased on direct palpation. Three weeks earlier he had been admitted to hospital and underwent investigations for epigastric pain. At that time, plain abdominal films and abdominal ultrasound were unremarkable. He was put on omeprazole and discharged home without definitive diagnosis. Past medical history was remarkable; he was a hepatitis B carrier and had an acute myocardial infarction six months before this admission.

On admission, the patient was febrile with a temperature 
of $39.9^{\circ} \mathrm{C}$, pulse of 100 beats $/ \mathrm{min}$ and regular blood pressure of 130/78 mmHg. Chest and cardiovascular examinations were unremarkable. Abdominal examination showed exquisite right upper quadrant tenderness and a positive Murphy's sign. Laboratory tests showed that his white blood cell count was $19.1 \times 10^{9} / \mathrm{L}$ with no left shift. Electrolytes and liver profiles were normal. Plain chest and abdominal films were unremarkable. With a presumptive diagnosis of acute cholecystitis, the patient was booked for a cholecystectomy. Preoperative ultrasound revealed a $3.5 \mathrm{~cm}$ abscess in the right lobe of the liver (Figure 3). Radiological findings recommended percutaneous drainage of the abscess. Because of the change in the ultrasound from normal just three weeks previously, the surgeon decided to perform an exploratory laparotomy.

The first part of the duodenum was densely adherent to the inferior surface of the liver. The gallbladder appeared normal. On dissecting the duodenum free from the liver, an abscess cavity within the inferior surface of the liver was entered. Following evacuation of the contents of the abscess cavity, a wooden toothpick $4 \mathrm{~cm}$ in length and $2 \mathrm{~mm}$ in diameter was found and removed from the abscess. Subsequent bacteriology on the abscess revealed a light growth of Klebsiella pneumoniae and of S viridans, and a scant growth of Candida albicans. The patient made a slow but uneventful recovery from his surgery.

\section{DISCUSSION}

Pyogenic hepatic abscesses are important but infrequent complications associated with significant morbidity and mortality resulting from ingested foreign bodies. Perforations of the bowel from ingested foreign bodies have been recognized as potential sources of liver abscesses. Infection spread via the biliary tree, portal system or arterial supply tends to cause multiple small abscesses, whereas direct extension and injury from foreign bodies usually produce large solitary abscesses (5). These symptoms are consistent with the findings in our cases. Each patient had a large solitary liver abscess caused by injury from wooden foreign bodies migrating from the gut. While the relationship between the physical nature of foreign bodies and the likelihood of perforation is not completely known, these circumstances suggest that long, hard and indigestible objects increase the risk of perforation.

Typically, foreign body perforations of the gastrointestinal tract that lead to hepatic abscesses are often not diagnosed preoperatively, as was the case in both our patients. The diagnosis of such conditions remains a challenging diagnostic problem for the clinician because it causes difficulty at each stage of the diagnostic process: history, physical examination, laboratory studies, imaging studies and laparotomy.

Foreign body ingestion occurs more commonly in children, the elderly or mentally impaired individuals. Other predisposing factors include impaired coordination of mastication, wearing dentures and intoxication due to substance abuse $(6,7)$. Obtaining a reliable history in these patients can be challenging. Furthermore, most patients often have a long asymptomatic interval between ingestion and the ap- pearance of symptoms. Only a few patients have recollection of swallowing foreign objects, which further impedes the diagnosis preoperatively. Our patients were similar to those at risk of foreign body ingestion: one was a mentally infirm patient and the other was an elderly adult who wore dentures. No history of swallowing foreign bodies was given in either case preoperatively. Only in retrospect, the second patient recalled swallowing a toothpick a month before surgery; he thought it had passed uneventfully. It is postulated that the pain this patient had experienced three weeks previously was a result of a perforation of his pylorus when he ingested a toothpick. This toothpick subsequently became embedded in the liver where it festered and resulted in the development of an abscess.

The clinical presentation is variable in patients who suffer from perforations of the gut with or without a liver abscess $(5,8)$. Some patients may present with nonspecific constitutional symptoms such as malaise, anorexia, nausea and vomiting. Others may manifest with generalized abdominal pain, mimicking an acute abdomen, that can be easily misdiagnosed as appendicitis, cholecystitis, diverticulitis, mechanical bowel obstruction or incarcerated hernia. This point was illustrated in the second case; our patient was mistakenly diagnosed with probable acute cholecystitis before the ultrasound. The presence of liver abscesses was also entirely unsuspected in both cases before the ultrasound or CT scan. In the second case, even after ultrasound identified the abscess, its etiology remained uncertain and the diagnosis of foreign body ingestion was established only after laparotomy.

The results of laboratory studies of patients with pyogenic liver abscesses typically show leukocytosis and anemia. Alkaline phosphatase, aspartate aminotransferase, alanine aminotransferase, serum bilirubin and erythrocyte sedimentation rate are frequently elevated $(5,8)$. In our cases, only the first patient showed an abnormal liver function test although both patients had liver abscesses. These findings suggest that routine laboratory tests may be helpful but not reliable in obtaining a correct etiological diagnosis.

The standard plain film is of limited assistance in suggesting the diagnosis because many foreign bodies (such as wooden material) are not radiopaque (9). Free intraperitoneal air suggests perforation but it gives no hint as to its etiology. Ultrasonography is now the preferred initial investigation in patients with acute right upper quadrant pain $(5,8,10)$. Ultrasonography can demonstrate abdominal abscesses and certain foreign bodies such as wooden materials. However, its utility is very operator-dependent; as illustrated in our second case, the toothpick was not prospectively diagnosed with ultrasonography, although on retrospective study the foreign body was visible as a tiny echogenic focus. The false negative ultrasound result was likely due to the lack of history available to the radiologist. Thus, the absence of a foreign body on ultrasound should not dissuade the clinician from the diagnosis. If a satisfactory ultrasonography cannot be obtained or if the clinician suspects the presence of abscesses associated with foreign body despite negative ultrasound, CT scanning is indicated. CT is particularly useful in 
patients in whom intestinal perforation or abdominal abscesses are suspected and in whom the clinical and standard radiological examinations are ambiguous (11). In these patients an attentive evaluation of the CT scan may identify any intra-abdominal abscess and unsuspected foreign objects in the abdominal cavity. However, ultrasound and CT scans are not meant to be screening tests. Their use should be considered following a proper history and physical examination to address specific clinical suspicions.

The cause of an acute abdomen may be impossible to determine preoperatively. In our first case, the intrahepatic abscess or gastrohepatic fistula was not identified by the initial exploratory laparotomy. Although it is not clear why the liver abscess or the gastrohepatic fistula was missed, it is speculated that the finding of other foreign bodies in the gut lowered the surgeon's index of suspicion for other coexisting pathologies. Thus, laparotomy - in particular without the aid of imaging studies or a high level of suspicion - even with direct visual and manual exploration, does not guarantee identification of a source. In our second case, a liver abscess was discovered by ultrasound, which facilitated the retrieval of the foreign body at laparotomy. Effective management in pyogenic liver abscesses consists of removal of the infective focus, proper drainage of the abscess and appropriate antibiotic therapy. A common initial antibiotic regimen includes penicillin, an aminoglycoside and clindamycin (5). In our first case, antibiotic therapy with gentamicin and clindamycin was given after the initial laparotomy. In retrospect, penicillin should have been added to the antibiotic regimen, especially given the positive culture for $S$ viridans. Percutaneous drainage of liver abscesses guided by an ultrasonic technique or a CT scan has gained widespread acceptance (12). However, the disadvantages of percutaneous drainage include the likelihood of the underlying etiology remaining undiagnosed and an increased risk of secondary procedure or operation $(13,14)$. In the second patient, the surgeon decided to perform an exploratory laparotomy instead of a percutaneous drainage procedure to increase the likelihood of determining the underlying etiology. Nevertheless, the recommended initial therapy for pyogenic hepatic abscess is percutaneous drainage $(8,12)$. Yet, if the abscess is caused by a foreign body, as in our cases, then open drainage and removal are required.

\section{CONCLUSIONS}

These cases show the need for clinicians to have a high index of suspicion for possible complications from ingested foreign bodies in managing unexplained liver abscesses. Ultrasound and CT scan can be very valuable diagnostic adjuncts in these difficult cases. Any patient with known factors risk for ingestion of foreign bodies should arouse suspicion and be investigated further.

ACKNOWLEDGEMENTS: The authors thank Drs S Gupta, $\mathrm{R}$ Gerencer and EK Schmidt for their contribution to this manuscript, and Drs Gerencer and Schmidt for their kind permission to publish the first case.

\section{REFERENCES}

1. Noel GJ, Karasic RB. Liver abscess following ingestion of a foreign body. Pediatr Infect Dis 1984;3:342-4.

2. Lawhorne TW, Schaff HV. Occult liver abscess and foreign body perforation of the bowel. Am Surg 1979;45:659-61.

3. Shaw PJ, Freeman JG. The antemortem diagnosis of pyogenic liver abscess due to perforation of the gut by a foreign body. Postgrad Med J 1983;59:455-6.

4. Pedersen VM, Geerdsen JP, Bartholdy J, et al. Foreign body perforation of the gastrointestinal tract with formation of liver abscess. Ann Chir Gynaecol 1986;75:245-6.

5. DeCock KM, Reynolds TB. Amebic and pyogenic liver abscess. In: Schiff L, Schiff ER, eds. Diseases of the Liver. Philadelphia: Lippincott Co, 1993.

6. McCanse DE, Kurchin A, Hinshaw JR. Gastrointestinal foreign bodies. Am J Surg 1981;142:335-7.

7. Schwartz JT, Graham DY. Toothpick perforation of the intestines. Ann Surg 1977;185:64-6.

8. Vukmir RB. Pyogenic hepatic abscess. Am Fam Phys 1993;47:1435-41.

9. Hewett PJ, Young JF. Toothpick injuries to the gastrointestinal tract. Aust NZ J Surg 1991;61:35-7.

10. Rioux M, Langis P. Sonographic detection of clinically unsuspected swallowed toothpicks and their gastrointestinal complications. J Clin Ultrasound 1994;22:483-90.

11. Struss JE, Bathazar EJ, Naidich DP. Jejunal peroration by a toothpick: CT demonstration. J Comput Assist Tomogr 1985;9:812-4.

12. Chu KM, Fan ST, Lai ECS, Lo CM, Wong J. Pyogenic liver abscess: An audit of experience over the past decade. Arch Surg 1996;131:148-52.

13. Branum GD, Tyson GS, Branum MA, Meyers WC. Hepatic abscess: Changes in etiology, diagnosis and management. Ann Surg 1990;212:655-22.

14. Bertel CK, van Heerden JA, Sheedy PF. Treatment of pyogenic hepatic abscesses, surgical vs percutaneous drainage. Arch Surg 1986;121:554-8. 


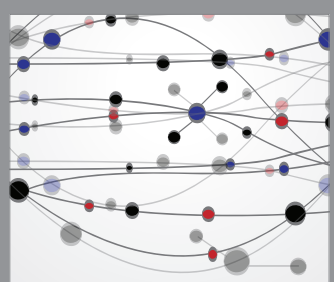

The Scientific World Journal
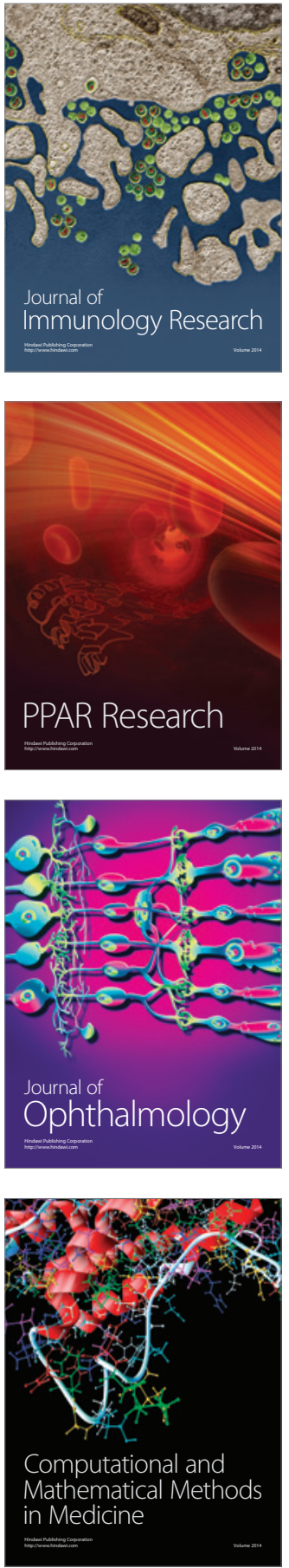

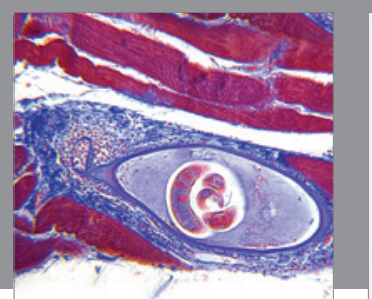

Gastroenterology Research and Practice

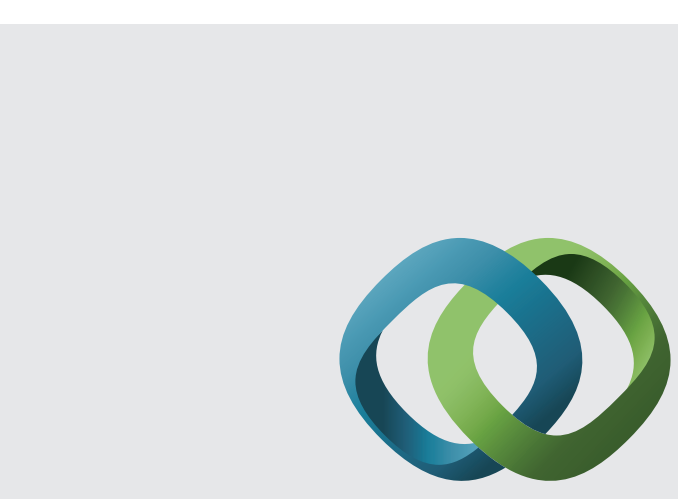

\section{Hindawi}

Submit your manuscripts at

http://www.hindawi.com
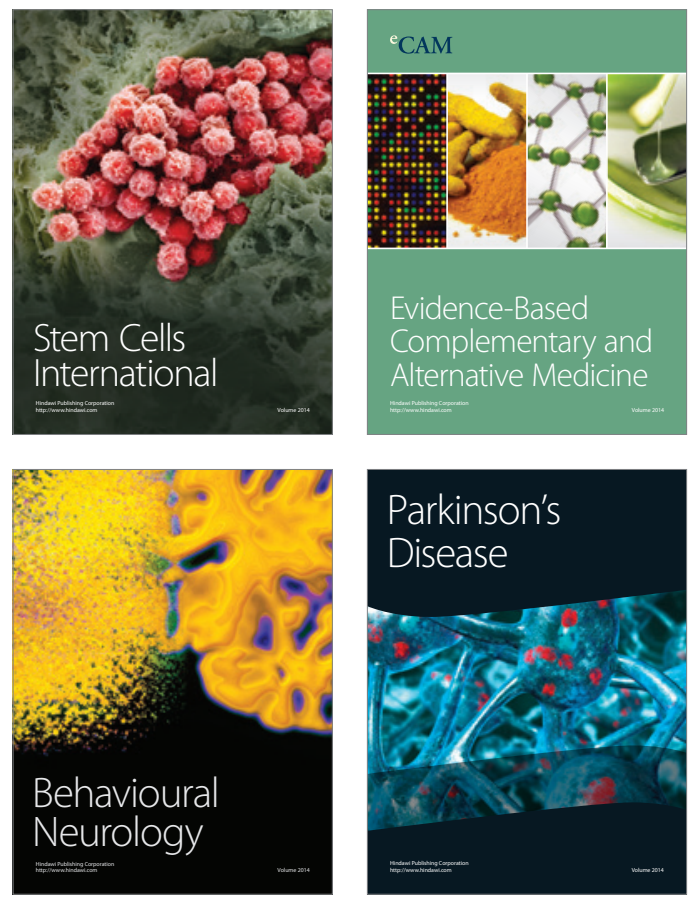
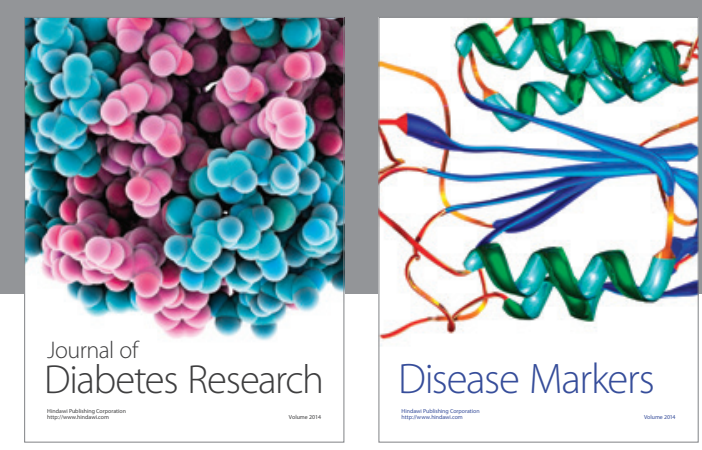

Disease Markers
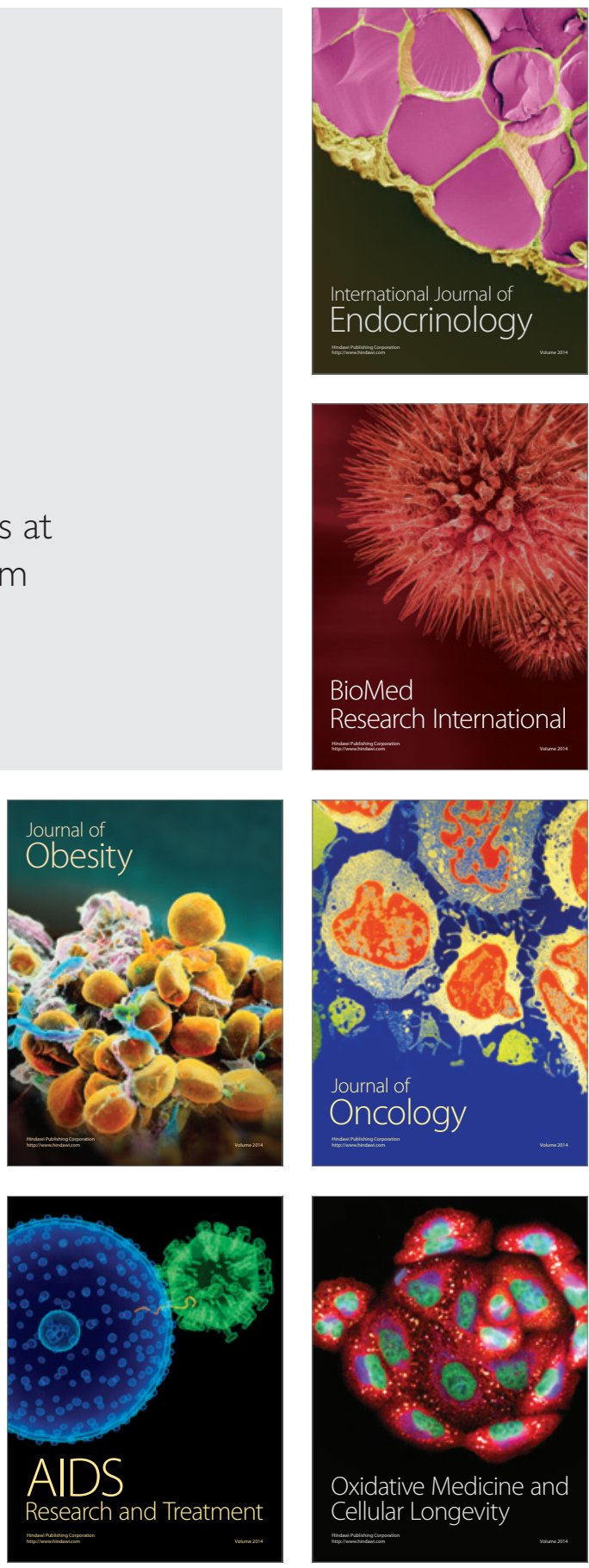